\title{
Effect of transition-metal substitutions on competing electronic transitions in the heavy-electron compound $\mathrm{URu}_{2} \mathrm{Si}_{2}$
}

\author{
Y. Dalichaouch and M. B. Maple \\ Department of Physics and Institute for Pure and Applied Physical Sciences, University of California, \\ San Diego, La Jolla, California 92093 \\ J. W. Chen \\ Department of Physics, National Taiwan University, Taipei, Taiwan \\ T. Kohara \\ Basic Research Laboratory, Himeji Institute of Technology, 2167 Shosha, Himeji, 671-22 Japan \\ C. Rossel \\ IBM Research Laboratory, Saumerstrasse 4, 8803 Ruschlikon, Zürich, Switzerland
}

M. S. Torikachvili

Department of Physics, San Diego State University, San Diego, California 92182-2222

A. L. Giorgi

Los Alamos National Laboratory, Materials Science and Technology Division, Los Alamos, New Mexico 87545

(Received 5 June 1989; revised manuscript received 19 October 1989)

\begin{abstract}
The moderately heavy-electron compound $\mathrm{URu}_{2} \mathrm{Si}_{2}$ is known to exhibit two electronic phase transitions at low temperatures, one to an antiferromagnetically ordered state at $T_{N}=17.5 \mathrm{~K}$ followed by another to a superconducting state at $T_{c} \sim 1.5 \mathrm{~K}$. The shape of the specific-heat anomaly at $T_{N}$, which is reminiscent of a second-order BCS-type mean-field transition, suggests the formation of a spin- or charge-density wave opening a gap over part of the Fermi surface. The effect of chemical substitution of the transition metals $M=R e, T c, O s, R h$, and $I r$ for $R u$ in $\mathrm{URu}_{2} \mathrm{Si}_{2}$ has been investigated by means of electrical resistivity, magnetic susceptibility, and specific-heat measurements in $\mathrm{URu}_{2-x} \mathrm{M}_{x} \mathrm{Si}_{2}$ for $x \leq 0.2$. The anomaly associated with the $17.5 \mathrm{~K}$ transition involves a very small magnetic entropy and is smeared out by very small concentrations of the $M$ substituent. An inverse correlation between $T_{c}$ and $T_{N}$ in the $\mathrm{Rh}$ - and Ir-doped materials for $x<0.01$ and in the Os-doped series for $x<0.1$ is consistent with the picture of two electronic transitions competing for states at the Fermi level.
\end{abstract}

\section{INTRODUCTION}

Heavy-electron rare-earth and actinide compounds have been of enormous interest over the past decade because of their remarkable normal- and superconductingstate properties. $^{1}$ At high temperatures (typically, $T \gtrsim 100 \mathrm{~K})$, the compounds exhibit localized $f$-electron behavior and the electrical resistivity is large, of the order of $100 \mu \Omega \mathrm{cm}$, whereas at low temperatures, the moments appear to be screened by the conduction electrons and the materials behave as strongly interacting Fermi liquids in which the quasiparticles (so-called heavy electrons) can be pictured as a superposition of localized $f$ electrons and conduction electrons. These quasiparticles have an effective mass $m^{*}$, inferred from the coefficient $\gamma$ of the electronic contribution to the specific heat, as high as several hundred times the free-electron mass. Some of these materials (e.g., $\mathrm{CeCu}_{2} \mathrm{Si}_{2}, \mathrm{UBe}_{13}, \mathrm{UPt}_{3}$, and $\mathrm{URu}_{2} \mathrm{Si}_{2}$ ) exhibit superconductivity below $1.5 \mathrm{~K}$, characterized by power-law dependences in the superconducting properties that are consistent with zeroes in the energy gap at points or lines on the Fermi surface.

Of this rich class of materials, the moderately heavyelectron compound $\mathrm{URu}_{2} \mathrm{Si}_{2}\left(m^{*} \approx 25 m_{e}\right)$ (Ref. 2-4) and, as recently reported, the heavy-electron compounds $\mathrm{UPt}_{3}$ (Ref. 5) and $\mathrm{CeCu}_{2.1} \mathrm{Si}_{2}$ (Ref. 6) show the coexistence of magnetism and superconductivity. The interplay between these two phenomena has been thoroughly studied in systems such as $R \mathrm{Rh}_{4} \mathrm{~B}_{4}$ and $R \mathrm{Mo}_{6} \mathrm{~S}_{8}$, where $R=$ rare earth. $^{7}$ In the latter compounds, magnetism and superconductivity are associated with two different subsets of electrons ( $4 f$ and $4 d$, respectively), whereas in the heavyelectron materials the magnetic and/or superconducting properties are determined by the same electrons ( $4 f$ for rare earth and $5 f$ for actinide compounds). Several heavy-electron compounds such as $\mathrm{U}_{2} \mathrm{Zn}_{17}, \mathrm{UCd}_{11}$, and $\mathrm{UCu}_{5}$ have been found to develop a magnetic ground state associated with the removal of part of the Fermi surface. $^{8-10}$ Alloy systems such as (U,Th)Pt $\mathbf{P}_{3}$ and $\mathrm{U}(\mathbf{P t}, \mathbf{P d})_{3}$ are also examples in which doping induces a 
long-range spin-density-wave (SDW) state $^{11,12}$ at the expense of superconductivity in $\mathrm{UPt}_{3}$. The compound $\mathrm{URu}_{2} \mathrm{Si}_{2}$ exhibits two distinct electronic transitions: bulk superconductivity below $1.5 \mathrm{~K}$ and a second transition at $T_{N}=17.5 \mathrm{~K}$. According to neutron scattering experiments on a $\mathrm{URu}_{2} \mathrm{Si}_{2}$ single crystal, the phase transition at $17.5 \mathrm{~K}$ is due to antiferromagnetic ordering with a very small moment on the $U$ site equal to $0.03 \pm 0.01 \mu_{B}$ and a modulation along the $(1,0,0)$ direction. ${ }^{13}$ The strongest evidence for a spin- or charge-density wave (SDW or $\mathrm{CDW})$ in $\mathrm{URu}_{2} \mathrm{Si}_{2}$ comes from specific-heat data. We pointed out ${ }^{4}$ that the anomaly $\delta C$ associated with the 17.5 $\mathrm{K}$ transition has a shape reminiscent of a secondorder BCS-type mean-field transition that can be described by the relation $\delta C=A \exp (-\Delta / T)$, where the activation energy $\Delta \approx 11 \mathrm{meV}$, and that the electronicspecific-heat coefficient $\gamma$ is reduced from $112 \mathrm{~mJ} / \mathrm{mol} \mathrm{K}^{2}$ above $T_{N}$ to $65.5 \mathrm{~mJ} / \mathrm{mol} \mathrm{K}$ above $1.5 \mathrm{~K}$. These observations along with the $\mathrm{Cr}$-like anomaly in the electrical resistivity and the small change in the magnetic susceptibility near $17.5 \mathrm{~K}$ led us to propose the establishment of a spin- or charge-density wave at $T_{N}$ that opens a gap over $\sim 40 \%$ of the Fermi surface. The absence of any structural transition in the vicinity of $17.5 \mathrm{~K}$ from $\mathrm{x}$-raydiffraction measurements that we made at various temperatures between 4 and $300 \mathrm{~K}$ suggests that the phase transition at $T_{N}$ is most likely associated with the establishment of a commensurate ${ }^{13}$ SDW state. Nuclear magnetic resonance, ${ }^{14}$ Hall effect ${ }^{15}$ and thermoelectric power ${ }^{16}$ measurements have been interpreted in favor of a $\mathrm{SDW}$ and/or $\mathrm{CDW}$ ground state in $\mathrm{URu}_{2} \mathrm{Si}_{2}$. Walter et al. reported the observation at $T=10 \mathrm{~K}$ of a $5.5 \mathrm{meV}$ gaplike magnetic excitation in the inelastic neutron scattering spectrum of a $\mathrm{URu}_{2} \mathrm{Si}_{2}$ polycrystalline sample. ${ }^{17}$

The purpose of this work is to obtain information about the competing electronic ground states in $\mathrm{URu}_{2} \mathrm{Si}_{2}$ by studying the effect of $4 d$ and $5 d$ transition-metal impurities on its physical properties. Chemical substitution of transition-metal $M$ ions for $\mathrm{Ru}$ is a direct way of varying the conduction-electron density and in turn modifying the topology of the Fermi surface, thus affecting the delicate balance between the electronic phase transitions at 1.5 and $17.5 \mathrm{~K}$. We have investigated a number of pseudoternary $\mathrm{URu}_{2-x} M_{x} \mathrm{Si}_{2}$ compounds, where $M=\mathrm{Tc}$, $\mathrm{Re}, \mathrm{Os}, \mathrm{Rh}$, and Ir for $0 \leq x \leq 0.2$, by means of electrical resistivity $\rho$, magnetic susceptibility $\chi$, and specific-heat $C$ measurements as functions of temperature $T$ in the range $70 \mathrm{mK} \leq T \leq 300 \mathrm{~K}$.

\section{EXPERIMENTAL DETAILS}

The polycrystalline $\mathrm{URu}_{2-x} M_{x} \mathrm{Si}_{2}$ samples were prepared by arc-melting stoichiometric amounts of the constituent elements together on a water-cooled-cooper hearth in a $\mathrm{Zr}$-gettered-ultrapure-argon atmosphere. The constituent elements had the following purities: U 99.9\%, Ru 99.9\%, Re 99.99\%, Rh 99.9\%, Os 99.9\%, Ir 99.99\%, Tc 99.9\%, and Si $99.999999 \%$. To control the stoichiometry for low-impurity concentrations $(x<0.05)$, appropriate amounts of $\mathrm{URu}_{2-\delta} T_{\delta} \mathrm{Si}_{2}$ with $\delta \leq 0.3$ and $\mathrm{URu}_{2} \mathrm{Si}_{2}$ master compounds were combined and melted together. The ingots were melted several times on each side to insure homogeneity, wrapped in Ta foil, sealed in a quartz tube in a 150 torr argon atmosphere, and annealed at $900^{\circ} \mathrm{C}$ for 8 days. X-ray-diffraction measurements revealed no impurity phases in all samples. Electrical resistivity measurements on bar-shaped specimens were made in a $\mathrm{He}^{4}$ cryostat using a four-lead ac impedance bridge operating at $16 \mathrm{~Hz}$. The dc magnetic susceptibility was measured in a field of $5 \mathrm{kOe}$ with a commercial superconducting quantum interference device (SQUID) susceptometer. The ac magnetic susceptibility was measured in a ${ }^{3} \mathrm{He}-{ }^{4} \mathrm{He}$ dilution refrigerator and the specific-heat data were taken using a semiadiabatic ${ }^{3} \mathrm{He}$ calorimeter with the standard heat-pulse method.

\section{RESULTS}

The ternary compounds $\mathrm{UM}_{2} \mathrm{Si}_{2}$ for $M=\mathrm{Ru}, \mathrm{Rh}$, Os, and Ir crystallize in the body-centered-tetragonal $\mathrm{ThCr}_{2} \mathrm{Si}_{2}$ structure, ${ }^{18}$ while $\mathrm{UTc}_{2} \mathrm{Si}_{2}$ and $\mathrm{URe}_{2} \mathrm{Si}_{2}$ form in a body-centered-cubic $\mathrm{URe}_{4} \mathrm{Si}_{6}$-type structure. ${ }^{19}$ All specimens of the $\mathrm{URu}_{2-x} M_{x} \mathrm{Si}_{2}$ series with $M=\mathrm{Tc}, \mathrm{Re}$, Os, $\mathrm{Rh}, \mathrm{Ir}$, Os, and $x \leq 0.2$ were found to form in the $\mathrm{ThCr}_{2} \mathrm{Si}_{2}$ structure. The composition dependence of the lattice parameters was investigated by means of x-ray analysis. The lattice parameters $a$ and $c$ determined by a least-squares fit of the high-angle lines are listed in Table I. Doping seems to decrease $a$ and increase $c$ in most cases except for Os, in which case an initial increase of $c$ is followed by a decrease above $x=0.02$.

The effect of transition-metal $M$ doping on the temperature dependence of the electrical resistivity $\rho$ of the $\mathrm{URu}_{2-x} M_{x} \mathrm{Si}_{2}(M=\mathrm{Rh}, \mathrm{Ir}, \mathrm{Os}$, and $\mathrm{Re})$ materials has been determined. The results are displayed in Figs. 1 and 2 as plots of the normalized electrical resistivity $\rho / \rho(300$ $\mathrm{K})$ versus temperature $T$. Figure 1 shows representative data for $M=$ Ir only. Also, for the sake of clarity, data for some values of $x$ are not shown. The three main regimes revealed above $20 \mathrm{~K}$ in the $\rho(T)$ data for $\mathrm{URu}_{2} \mathrm{Si}_{2}$ (Fig. 1) are (1) an increase in $\rho$, as $T$ is lowered from 300 to $\sim 80 \mathrm{~K}$, which is associated with the Kondo scattering of the conduction electrons by independent spins; (2) the development of a broad maximum in $\rho(T)$ centered near $T_{M} \approx 79 \mathrm{~K}$; and (3) a rapid decrease in $\rho$ as the temperature is lowered further. This latter feature is associated with the onset of coherent scattering of the conduction electrons by the periodically distributed $U$ ions. Below $20 \mathrm{~K}$, a narrow peak associated with the SDW transition develops in the vicinity of $T_{N}=17.5 \mathrm{~K}$. The value of $T_{N}$ is defined by the minimum in the $(1 / \rho)(d \rho / d T)$ versus $T$ curve as plotted for $\mathrm{URu}_{2-x} \mathrm{Rh}_{x} \mathrm{Si}_{2}$ in Fig. 3. Finally, there is a transition to the superconducting state below $T_{c} \approx 1.5 \mathrm{~K}$.

The effect of doping on the shape of $\rho(T)$ is minimal above $T_{M}$. The temperature coefficient of resisitivity remains negative as in $U \mathrm{Uu}_{2} \mathrm{Si}_{2}$ but of lower (Ir and $\mathrm{Re}$ ) or higher ( $R h$ and Os) magnitude than for the pure compound, which is qualitatively consistent with a respective 
TABLE I. Lattice parameters for $\mathrm{URu}_{2-x} M_{x} \mathrm{Si}_{2}$ alloys determined from x-ray-diffraction measurements at $300 \mathrm{~K}$.

\begin{tabular}{lcccc}
\multicolumn{1}{c}{ Compound } & $a(\AA)$ & $c(\AA)$ & $c / a$ & $V\left(\AA^{3}\right)$ \\
\hline $\mathrm{URu}_{2} \mathrm{Si}_{2}$ & 4.142 & 9.559 & 2.308 & 164.00 \\
$\mathrm{URu}_{1.99} \mathrm{Rh}_{0.005} \mathrm{Si}_{2}$ & 4.133 & 9.584 & 2.319 & 163.71 \\
$\mathrm{URu}_{1.99} \mathrm{Rh}_{0.01} \mathrm{Si}_{2}$ & 4.132 & 9.598 & 2.323 & 163.87 \\
$\mathrm{URu}_{1.98} \mathrm{Rh}_{0.02} \mathrm{Si}_{2}$ & 4.125 & 9.594 & 2.326 & 163.25 \\
$\mathrm{URu}_{1.95} \mathrm{Rh}_{0.05} \mathrm{Si}_{2}$ & 4.125 & 9.621 & 2.332 & 163.71 \\
$\mathrm{URu}{ }_{1.9} \mathrm{Rh}_{0.1} \mathrm{Si}_{2}$ & 4.142 & 9.624 & 2.324 & 165.11 \\
$\mathrm{URu}_{1.99} \mathrm{Os}_{0.01} \mathrm{Si}_{2}$ & 4.131 & 9.577 & 2.318 & 163.43 \\
$\mathrm{URu}_{1.98} \mathrm{Os}_{0.02} \mathrm{Si}_{2}$ & 4.125 & 9.594 & 2.326 & 163.25 \\
$\mathrm{URu}_{1.97} \mathrm{Os}_{0.03} \mathrm{Si}_{2}$ & 4.132 & 9.574 & 2.317 & 163.46 \\
$\mathrm{URu}_{1.95} \mathrm{Os}_{0.05} \mathrm{Si}_{2}$ & 4.133 & 9.567 & 2.315 & 163.42 \\
$\mathrm{URu}{ }_{1.9} \mathrm{Os}_{0.1} \mathrm{Si}_{2}$ & 4.117 & 9.544 & 2.318 & 161.77 \\
$\mathrm{URu}_{1.99} \mathrm{Ir}_{0.01} \mathrm{Si}_{2}$ & 4.132 & 9.504 & 2.300 & 162.27 \\
$\mathrm{URu}_{1.98} \mathrm{Ir}_{0.02} \mathrm{Si}_{2}$ & 4.112 & 9.587 & 2.331 & 162.10 \\
$\mathrm{URu}_{1.95} \mathrm{Ir}_{0.05} \mathrm{Si}_{2}$ & 4.132 & 9.618 & 2.328 & 164.21 \\
$\mathrm{URu}_{1.9} \mathrm{Re}_{0.1} \mathrm{Si}_{2}$ & 4.142 & 9.550 & 2.306 & 163.84 \\
$\mathrm{URu}_{1.8} \mathrm{Re}_{0.2} \mathrm{Si}_{2}$ & 4.138 & 9.583 & 2.316 & 164.09 \\
$\mathrm{URu}_{1.88} \mathrm{Tc}_{0.12} \mathrm{Si}_{2}$ & 4.134 & 9.571 & 2.315 & 163.57 \\
\hline \hline
\end{tabular}

increase or decrease of the Kondo temperature $T_{K}$. The temperature $T_{M}$ of the maximum in $\rho(T)$ is invariably shifted to higher temperatures as indicated in Table II, except for $\mathrm{Rh}$, where it decreases with increasing $x$. Below $T_{M}$, all $\rho(T)$ curves show coherent scattering of the conduction electrons. The most dramatic effect of the transition-metal substitution is on the phase transition at $T_{N}$ as shown in Fig. 2. The partial replacement of $\mathrm{Ru}$ with nonisoelectronic $\mathrm{Ir}, \mathrm{Re}$, or $\mathrm{Rh}$ atoms results in a decrease of $T_{N}$. In addition, the anomaly gets smeared out for low-impurity concentrations, especially in the case of Ir substitution, where it is completely suppressed at $x=0.03$. The effect of $\mathrm{Rh}$ substitution is less destructive: the anomaly is considerably spread out but still noticeable below $10 \mathrm{~K}$ for $x=0.05$. Incorporation of $\mathrm{Re}$ seems to have the least effect on the shape of the feature at $T_{N}$;

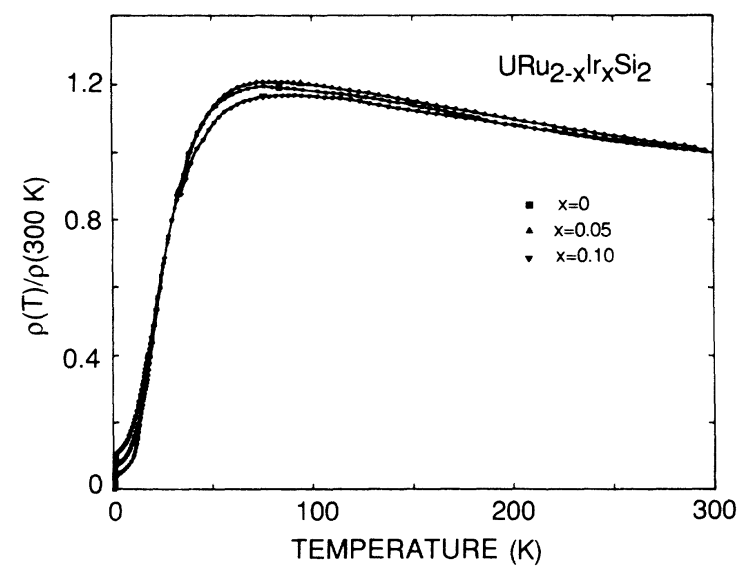

FIG. 1. Normalized electrical resistivity $\rho(T) / \rho(300 \mathrm{~K})$ vs temperature $T$ data for $\mathrm{URu}_{2-x} \mathrm{Ir}_{x} \mathrm{Si}_{2}$ compounds with various compositions $x$. the transition is clearly seen for $x=0.05$, although it is broadened somewhat and is completely removed only for $x=0.1$. In contrast, the substitution of isoelectronic Os for $\mathrm{Ru}$ results in a completely different behavior: $T_{N}$ increases with $x$, first slowly for $x \leq 0.04$ and then more rapidly for $x>0.04$, reaching the value of $19.8 \mathrm{~K}$ for $x=0.1$, at which point the transition is enormously broadened. Assuming that the resistivity of $\mathrm{URu}_{2} \mathrm{Si}_{2}$ at $300 \mathrm{~K}$ does not substantially change upon substitution (at least up to $x=0.01),{ }^{20}$ we can infer from the $\rho(300$ $\mathrm{K}) / \rho(2 \mathrm{~K})$ values listed in Table II that the increase of the residual resistivity $\rho_{0}$ at $T \gtrsim T_{c}$ depends strongly on the

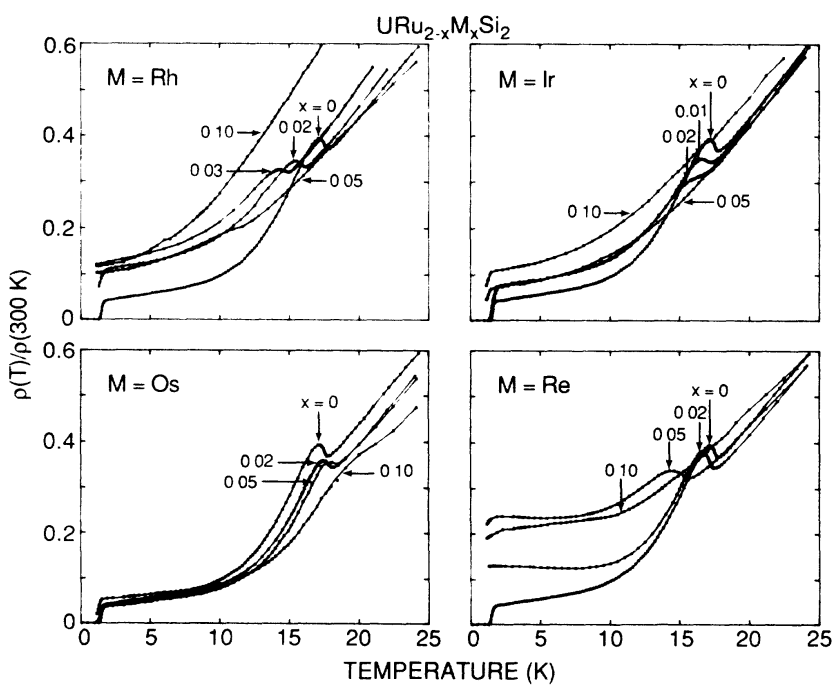

FIG. 2. Normalized low-temperature electrical resistivity $\rho(T) / \rho(300 \mathrm{~K})$ vs temperature $T$ data for $\mathrm{URu}_{2-x} M_{x} \mathrm{Si}_{2}$ compounds with various compositions $x$. For clarity, data for some compositions are not shown. 


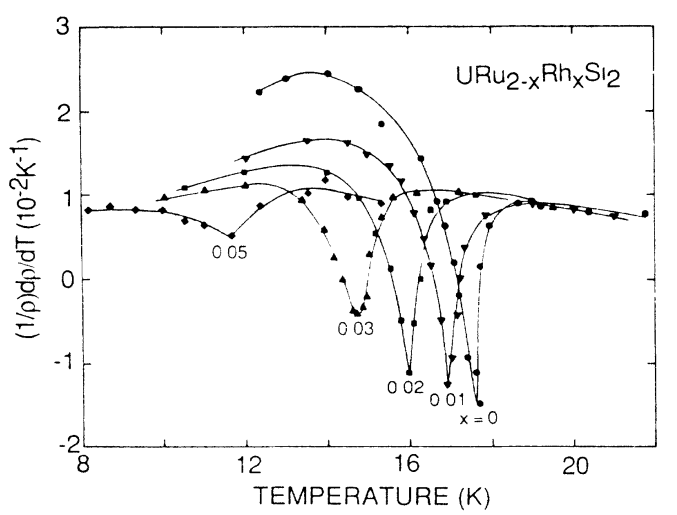

FIG. 3. Temperature coefficient of electrical resistivity $(1 / \rho)(d \rho / d T)$ of $\mathrm{URu}_{2-x} \mathrm{Rh}_{x} \mathrm{Si}_{2}$ vs temperature $T$ for selected compositions $x$. The SDW transition temperature $T_{N}$ is defined as the temperature corresponding to the minimum in $(1 / \rho)(d \rho / d T)$.

solute element and is the largest for Re. The incorporation of $\mathrm{Rh}$ and $\mathrm{Ir}$ atoms induces a moderate increase in $\rho_{0}$, whereas Os atoms do not alter it significantly.

Shown in Figs. 4 and 5 are representative results of $\chi(T)$ measurements for $M=O$ s and $\mathrm{Ir}$, respectively. The magnetic subceptibility exhibits single-ion Kondo behavior below $300 \mathrm{~K}$, followed by a rounded maximum near $55 \mathrm{~K}$ that correlates with a similar maximum in the $\rho(T)$ data. However, the maximum in $\chi(T)$ occurs at a temperature $20 \mathrm{~K}$ lower than that in $\rho(T)$. The magnetic transition is characterized by a sudden but small decrease of $\chi(T)$ near $T_{N} \approx 17.5 \mathrm{~K}$, defined as the inflection point of the anomaly. The Curie-Weiss behavior above $T_{M}$ is not influenced by alloying, and no clear trend is observed for $\mathrm{Rh}$ and Ir substitutions for the compositions investigated. In contrast, the magnetic susceptibility above 20 $\mathrm{K}$ increases in the $\mathrm{URu}_{2-x} \mathrm{Os}_{x} \mathrm{Si}_{2}$ materials. The effect on $T_{N}$ of transition-metal doping inferred from the $\chi(T)$ data is similar to that determined from the $\rho(T)$ data:

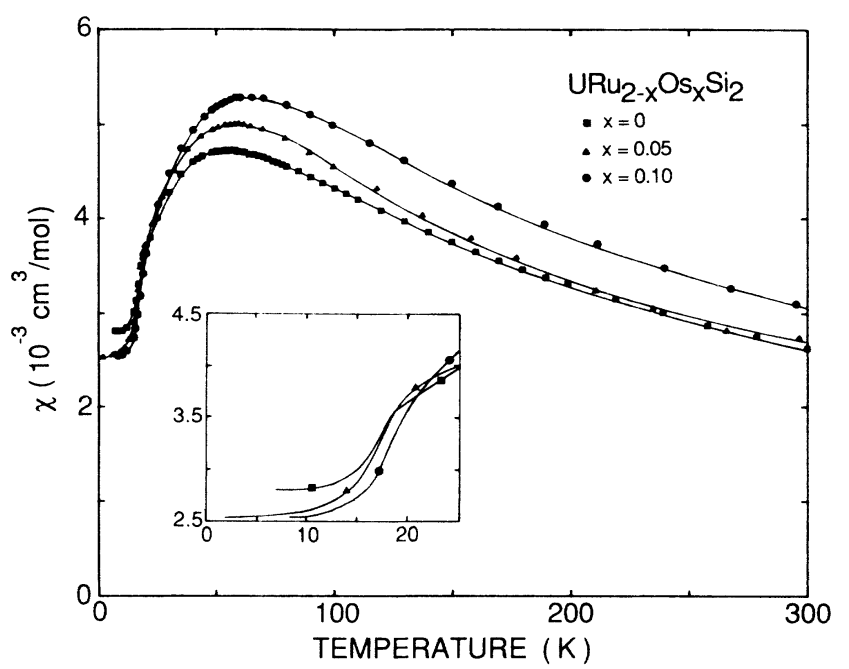

FIG. 4. Magnetic susceptibility $\chi$ vs temperature $T$ for $\mathrm{URu}_{2-x} \mathrm{Os}_{x} \mathrm{Si}_{2}$ compounds with various compositions $x$. Inset: $\chi$ vs $T$ for $T \leq 25 \mathrm{~K}$.

broadening of the anomaly and a decrease (increase) of $T_{N}$ for $M=\mathrm{Rh}, \mathrm{Ir}$, and $\operatorname{Re}(\mathrm{Os})$ as shown in the insets of Figs. 4 and 5. The substitution of Tc at $x=0.05$, as revealed by $\chi(T)$ data, depresses $T_{N}$ to $16.2 \mathrm{~K}$, a value close to that of $\mathrm{URu}_{1.95} \mathrm{Re}_{0.05} \mathrm{Si}_{2}$. It is interesting to note that in the Os-doped samples, not only $T_{N}$, but also the magnitude of the susceptibility change associated with the transition, increases with $x$. With the exception of the $\mathrm{URu}_{2-x} \mathrm{Re}_{x} \mathrm{Si}_{2}$ materials, the susceptibility reaches a plateau at lower temperatures and $\chi(0) \approx(2.5-3.0)$ $\times 10^{-3} \mathrm{~cm}^{3} / \mathrm{mol}$ is of the same order as in $\mathrm{URu}_{2} \mathrm{Si}_{2}$.

The magnetic behavior of the $\mathrm{Re}$ - and Tc-doped materials is anomalous. For $\mathrm{URu}_{2-x} \mathrm{Re}_{x} \mathrm{Si}_{2}$ in the range $0 \leq x \leq 0.1$, the susceptibility at all temperatures increases and is accompanied by an upturn at low temperatures. With a further increase of $x$, the susceptibility at $300 \mathrm{~K}$ decreases; however, as the temperature is lowered

TABLE II. Characteristic temperatures $T_{M}$, determined from magnetic susceptibility $\chi$ and electrical resistivity $\rho$ measurements, $0 \mathrm{~K}$ extrapolated values of the electronic specific heat coefficient $\gamma$, and residual resistivity ratios $\rho(300 \mathrm{~K}) / \rho(2 \mathrm{~K})$ for selected samples in the $\mathrm{URu}_{2-x} M_{x} \mathrm{Si}_{2}$ alloy systems.

\begin{tabular}{|c|c|c|c|c|}
\hline Compound & $\begin{array}{c}(\mathbf{K}) \\
\left(T_{M}\right)_{\chi}\end{array}$ & $\begin{array}{c}(\mathrm{K}) \\
\left(T_{M}\right)_{\rho}\end{array}$ & $\begin{array}{c}\mathrm{mJ} / \mathrm{mol} \mathrm{K}^{2} \\
\gamma\end{array}$ & $\rho(300 \mathbf{K}) / \rho(2 \mathbf{K})$ \\
\hline $\mathrm{URu}_{2} \mathrm{Si}_{2}$ & 55 & 79 & 65.5 & 24.6 \\
\hline $\mathrm{URu}_{1.98} \mathrm{Rh}_{0.02} \mathrm{Si}_{2}$ & 60 & 79 & & 9.1 \\
\hline $\mathrm{URu}_{1.95} \mathrm{Rh}_{0.05} \mathrm{Si}_{2}$ & 51.8 & & & 9.6 \\
\hline $\mathrm{URu}_{1.9} \mathbf{R h}_{0.1} \mathrm{Si}_{2}$ & 49.4 & 69 & 105 & 8.2 \\
\hline $\mathrm{URu}_{1.95} \mathrm{Os}_{0.05} \mathrm{Si}_{2}$ & 57.2 & 86 & & 26.4 \\
\hline $\mathrm{URu}_{1.9} \mathrm{Os}_{0.1} \mathrm{Si}_{2}$ & 66.4 & 92 & & 17.9 \\
\hline $\mathrm{URu}_{1.95} \mathrm{Ir}_{0.05} \mathrm{Si}_{2}$ & 55 & 79.5 & & 13.5 \\
\hline $\mathrm{URu}_{1.9} \mathrm{Ir}_{0.1} \mathrm{Si}_{2}$ & 60 & 90.5 & & 9.1 \\
\hline $\mathrm{URu}_{1.95} \mathrm{Re}_{0.05} \mathrm{Si}_{2}$ & 59.5 & 90 & & 4.2 \\
\hline $\mathrm{URu}_{1.9} \mathrm{Re}_{0.1} \mathrm{Si}_{2}$ & 59.1 & 89 & 70.5 & 4.9 \\
\hline $\mathrm{URu}_{1.8} \mathrm{Re}_{0.2} \mathrm{Si}_{2}$ & 53.9 & 88.5 & & 4.4 \\
\hline $\mathrm{URu}_{1.95} \mathrm{Tc}_{0.05} \mathrm{Si}_{2}$ & 50 & & & \\
\hline
\end{tabular}




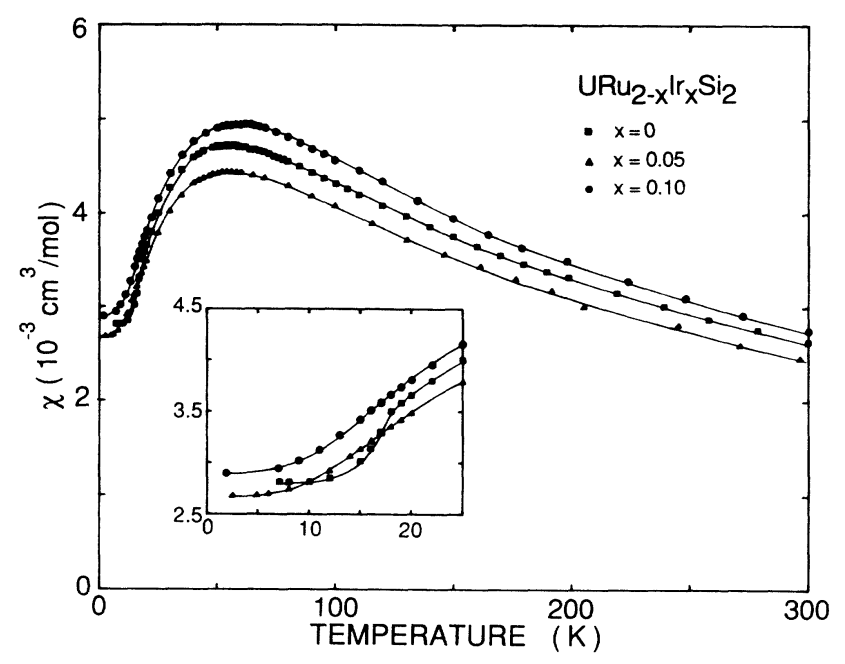

FIG. 5. Magnetic susceptibility $\chi$ vs temperature $T$ for $\mathrm{URu}_{2-x} \mathrm{Ir}_{x} \mathrm{Si}_{2}$ compounds with various compositions $x$. Inset: $\chi$ vs $T$ for $T \leq 25 \mathrm{~K}$.

there is a crossover and the system again becomes more magnetic, displaying a large upturn in $\chi(T)$ below $10 \mathrm{~K}$. A linear extrapolation to $0 \mathrm{~K}$ yields $\chi(0) \approx 6 \times 10^{-3}$ $\mathrm{cm}^{3} / \mathrm{mol}$, a value two times larger than that of $\mathrm{URu}_{2} \mathrm{Si}_{2}$. The changes in $\chi(T)$ are greater in the $\mathrm{URu}_{2-x} \mathrm{Tc}_{x} \mathrm{Si}_{2}$ series. For $x \leq 0.12$, the susceptibility decreases by $\sim 30 \%$ at $300 \mathrm{~K}$ and shows a very weak temperature dependence. For $x=0.05$, the rounded maximum shows significant broadening and is virtually nonexistent for $\mathrm{URu}_{1.88} \mathrm{Tc}_{0.12} \mathrm{Si}_{2}$. This anomalous behavior of $\chi(T)$ appears to be the precursor of the ferromagnetic ordering we recently discovered in the $\mathrm{URu}_{2-x} \mathrm{Re}_{x} \mathrm{Si}_{2}$ and $\mathrm{URu}_{2-x} \mathrm{Tc}_{x} \mathrm{Si}_{2}$ systems in the composition range $0.4 \lesssim x \lesssim 1.4 .^{21}$

Inductive measurements of the superconducting transition temperature $T_{c}$ for the $\mathrm{URu}_{2-x} M_{x} \mathrm{Si}_{2}$ materials yielded values slightly lower than those obtained from electrical resistivity measurements. In this work, $T_{c}$ is

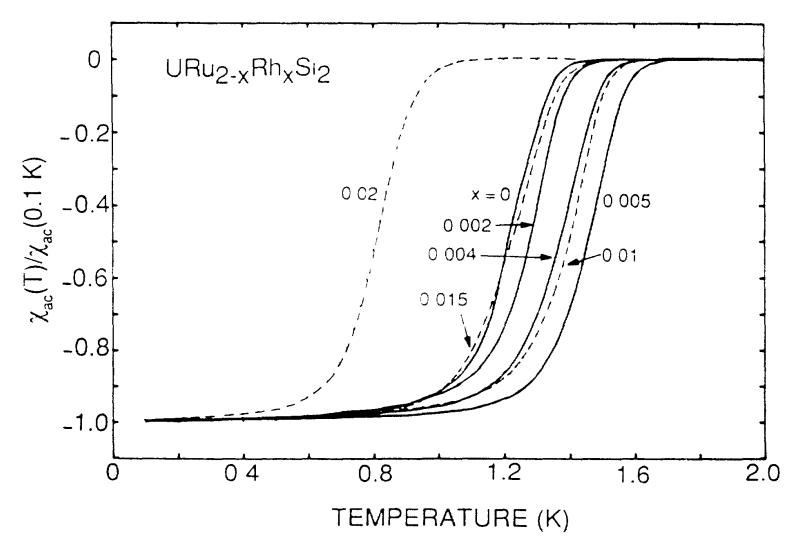

FIG. 6. Magnetic susceptibility $\chi_{\text {ac }}$ vs temperature $T$ for $\mathrm{URu}_{2-x} \mathrm{Rh}_{x} \mathrm{Si}_{2}$ compounds with various compositions $x \leq 0.02$ in zero applied magnetic field. defined as the temperature where the magnitude of $\chi_{\mathrm{ac}}$ has decreased by $50 \%$ of the total change associated with the superconducting transition. Normalized $\chi_{\mathrm{ac}} / \chi_{\mathrm{ac}}(0.1$ $\mathrm{K})$ versus $T$ data are displayed in Fig. 6 for $M=\mathrm{Rh}$. In general, the data reveal a progressive broadening of the superconducting transition as a function of impurity concentration $x$, especially in the Ir-substituted samples for $x>0.03$ (data not shown). The substitution of transition metals $M=\mathrm{Re}$, and Os for $\mathrm{Ru}$, leads to a rapid depression of $T_{c}$, particularly in the case of $\mathrm{Re}$, where $T_{c}$ drops to $0.23 \mathrm{~K}$ for $x=0.01$. In the case of Os substitutions, $T_{c}$ decreases more gradually and is equal to $0.80 \mathrm{~K}$ for $x=0.03$. The dependence of $T_{c}$ on $\mathrm{Rh}$ and $\mathrm{Ir}$ composition $x$ is similar, $T_{c}$ first increases, passes through a maximum, and then decreases with further increase of $x$. However, $T_{c}$ cannot be determined accurately beyond $x=0.04$ in the $\mathrm{URu}_{2-x} \mathrm{Ir}_{x} \mathrm{Si}_{2}$ series because of the broadening of the transition widths and the scatter in the $T_{c}(x)$ data. Doping with $\mathrm{Rh}$ at the level $x=0.03$ results in a $T_{c}$ that is considerably depressed, with a superconducting onset below $0.4 \mathrm{~K}$. Also, a sample of $\mathrm{URu}_{1.95} \mathrm{Tc}_{0.05} \mathrm{Si}_{2}$ was checked and found nonsuperconducting above $70 \mathrm{mK}$.

Specific heat $C$ divided by $T$ versus $T^{2}$ data below $20 \mathrm{~K}$ are shown in Fig. 7 for $\mathrm{URu}_{2} \mathrm{Si}_{2}, \mathrm{URu}_{1.9} \mathrm{Re}_{0.1} \mathrm{Si}_{2}$, and $\mathrm{URu}_{1.9} \mathrm{Rh}_{0.1} \mathrm{Si}_{2}$. The substitution of 5 at. \% Re or $\mathrm{Rh}$ for $\mathrm{Ru}$ in $\mathrm{URu} \mathrm{u}_{2} \mathrm{Si}_{2}$ hardly affects the specific heat above $18 \mathrm{~K}$ but suppresses the anomaly at $T_{N}$ almost entirely. The jump at $T_{N}$ is considerably reduced, broadened and transformed into a rounded maximum at $\sim 12.6 \mathrm{~K}$ for $\mathrm{URu}_{1.9} \mathrm{Re}_{0.1} \mathrm{Si}_{2}$ and an extremely broad shoulder below $10 \mathrm{~K}$ for $\mathrm{URu}_{1.9} \mathrm{Rh}_{0.1} \mathrm{Si}_{2}$. The normal-state specific heat of $\mathrm{URu}_{2} \mathrm{Si}_{2}$ between 2 and $18 \mathrm{~K}$ can be described by

$$
C=\gamma T+\beta T^{3}+A \exp (-\Delta / T),
$$

where $\gamma T, \beta T^{3}$, and $A \exp (-\Delta / T)$ represent, respectively, the electronic, lattice, and SDW-state contributions. Above $T_{N}$, the data can be fitted to $C=\gamma^{\prime} T+\beta^{\prime} T^{3}$ with $\gamma^{\prime}=112 \mathrm{~mJ} / \mathrm{mol} \mathrm{K}^{2}$ and $\beta^{\prime}=0.382 \mathrm{~mJ} / \mathrm{mol} \mathrm{K}^{2}\left(\Theta_{D}=294\right.$ $\mathrm{K})$. A least-squares fit of the SDW contribution to the specific heat to

$$
\delta C=A \exp (-\Delta / T)
$$

in a manner described in Ref. 4 yields $A=989$ $\mathrm{J} / \mathrm{mol} \mathrm{K}=490 \gamma T_{N}$ and $\Delta=129 \mathrm{~K}=7.2 T_{N}$. The jump in specific heat at $17.5 \mathrm{~K}$ is equal to $5.8 \mathrm{~J} / \mathrm{mol} \mathrm{K}=2.9 \gamma T_{N}$, and the entropy $\Delta S=\int_{0}^{T_{N}}(\delta C / T) d T$ is estimated to be $980 \mathrm{~mJ} / \mathrm{mol} \mathrm{K}=0.170 k_{B} \ln 2$ per formula unit. From the ratio of the ungapped $\gamma$ above $18 \mathrm{~K}$ to the low temperature $\gamma=65.5 \mathrm{~mJ} / \mathrm{mol} \mathrm{K}^{2}$ obtained by extrapolating the normal-state data above $1.5 \mathrm{~K}$ to $T=0$, it has been estimated that $40 \%$ of the Fermi surface is removed at $T_{N}$ by the transition to the SDW state.

The jump in the specific heat at $T_{N} \approx 13 \mathrm{~K}$ in $\mathrm{URu}_{1.9} \mathrm{Re}_{0.1} \mathrm{Si}_{2}$ is equal to $1.3 \mathrm{~J} / \mathrm{mol} \mathrm{K}=1.1 \gamma T_{N}$, a value much smaller than in pure $\mathrm{URu}_{2} \mathrm{Si}_{2}$. The data above 13 $\mathrm{K}$ can be described by $C=\gamma^{\prime} T+\beta^{\prime} T^{3}+\alpha^{\prime} T^{5}$ with $\gamma^{\prime}=90$ $\mathrm{mJ} / \mathrm{mol} \mathrm{K}^{2}, \beta^{\prime}=0.345 \mathrm{~mJ} / \mathrm{mol} \mathrm{K}{ }^{4}\left(\Theta_{D}=304 \mathrm{~K}\right)$, and $\alpha^{\prime}=4.91 \times 10^{-4} \mathrm{~mJ} / \mathrm{mol} \mathrm{K}^{6}$. Following the same method 


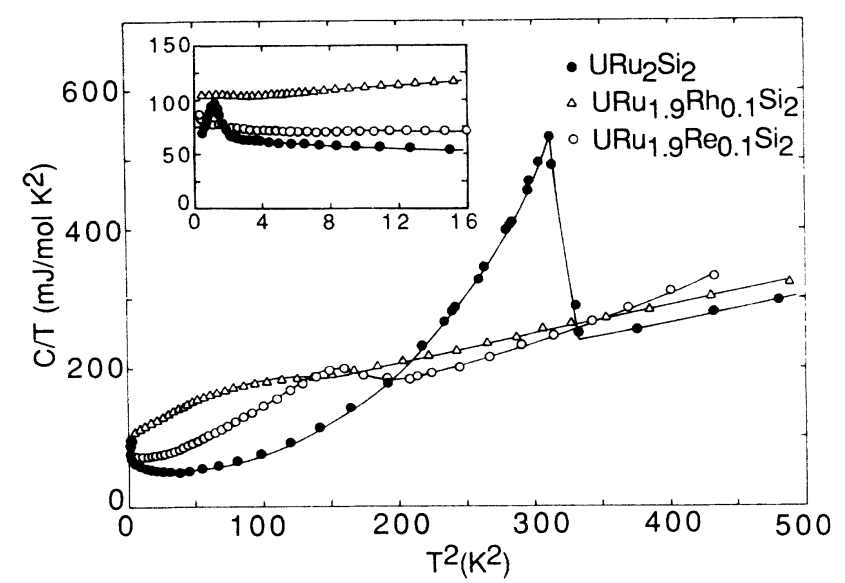

FIG. 7. Specific heat $C$ divided by temperature $T$ vs $T^{2}$ for $\mathrm{URu}_{2} \mathrm{Si}_{2}$ and $\mathrm{URu}_{1.9} \mathrm{M}_{0.1} \mathrm{Si}_{2}$ with $M=\mathrm{Rh}$ and $\mathrm{Re}$. Inset: $C / T$ vs $T^{2}$ for $T \leq 4 \mathrm{~K}$.

used for $\mathrm{URu}_{2} \mathrm{Si}_{2}$, a least-squares fit of the SDW part of the specific heat to Eq. (2) yields $A=6.1 \mathrm{~J} / \mathrm{mol} \mathrm{K}$ and $\Delta=17 \mathrm{~K}$. In this case, the instability removes only $\sim 25 \%$ of the Fermi surface and the entropy associated with the transition is estimated to be 290 $\mathrm{mJ} / \mathrm{mol} \mathrm{K}=0.05 k_{B} \quad \ln 2$. The specific heat of $\mathrm{URu}_{1.9} \mathrm{Rh}_{0.1} \mathrm{Si}_{2}$ is well described between 14 and $22 \mathrm{~K}$ by $C=\gamma^{\prime} T+\beta^{\prime} T^{3}$ with $\gamma^{\prime}=130 \mathrm{~mJ} / \mathrm{mol} \mathrm{K}^{2}$ and $\beta^{\prime}=0.403$ $\mathrm{mJ} / \mathrm{mol} \mathrm{K}^{4}\left(\Theta_{D}=289 \mathrm{~K}\right)$. The gap contribution $\delta C$ can still be fitted to Eq. (2) with $A=1.2 \mathrm{~J} / \mathrm{mol} \mathrm{K}$ and $\Delta=4$ $\mathrm{K}$. Less than $20 \%$ of the Fermi surface is removed in this case, and the transition, although very broad, still involves an entropy of about $270 \mathrm{~mJ} / \mathrm{mol} \mathrm{K}$, close to that of $\mathrm{URu}_{1.9} \operatorname{Re}_{0.1} \mathrm{Si}_{2}$. The low-temperature data displayed in the inset of Fig. 7 reveal an upturn below $1 \mathrm{~K}$ in the Redoped material that correlates with a similar upturn in the magnetic susceptibility. A linear extrapolation of the $C / T$ versus $T^{2}$ from above 1 to $0 \mathrm{~K}$ yields an electronicspecific-heat coefficient $\gamma=71 \mathrm{~mJ} / \mathrm{mol} \quad \mathrm{K}^{2}$ for $\mathrm{URu}_{1.9} \mathrm{Re}_{0.1} \mathrm{Si}_{2}$ and $105 \mathrm{~mJ} / \mathrm{mol} \mathrm{K}{ }^{2}$ for $\mathrm{URu}_{1.9} \mathrm{Rh}_{0.1} \mathrm{Si}_{2}$.

\section{DISCUSSION}

The magnetic transition at $T_{N}$, associated with the development of a SDW state is strongly affected by transition-metal $M$ substitution at the Ru sites. The transition is smeared out by very small impurity concentrations. A plot summarizing $T_{c}(x)$ and $T_{N}(x)$ for all of the transition-metal substitutions investigated is shown in Fig. 8. On the basis of chemical pressure arguments alone, one would expect that the effect of substitution on $T_{c}$ or $T_{N}$ would be similar for all solutes since they all have metallic radii larger than that of $\mathrm{Ru}\left(r_{\mathrm{Ru}}=1.339 \AA\right.$, $r_{\mathrm{Rh}}=1.345 \AA, r_{\mathrm{Re}}=1.375 \AA, r_{\mathrm{Tc}}=1.360 \AA, r_{\mathrm{Os}}=1.353$ $\AA$, and $r_{\mathrm{Ir}}=1.357 \AA$ ). ${ }^{22}$ However, the fact that $T_{N}$ increases with $x$ for $M=O$ s and decreases with $x$ for $M=\mathrm{Rh}, \mathrm{Re}, \mathrm{Tc}$, and $\mathrm{Ir}$ in the range $0 \leq x \leq 0.1$ indicates that chemical pressure is not the prime factor influencing $T_{N}$.

The large spacing between $U$ atoms in heavy-electron

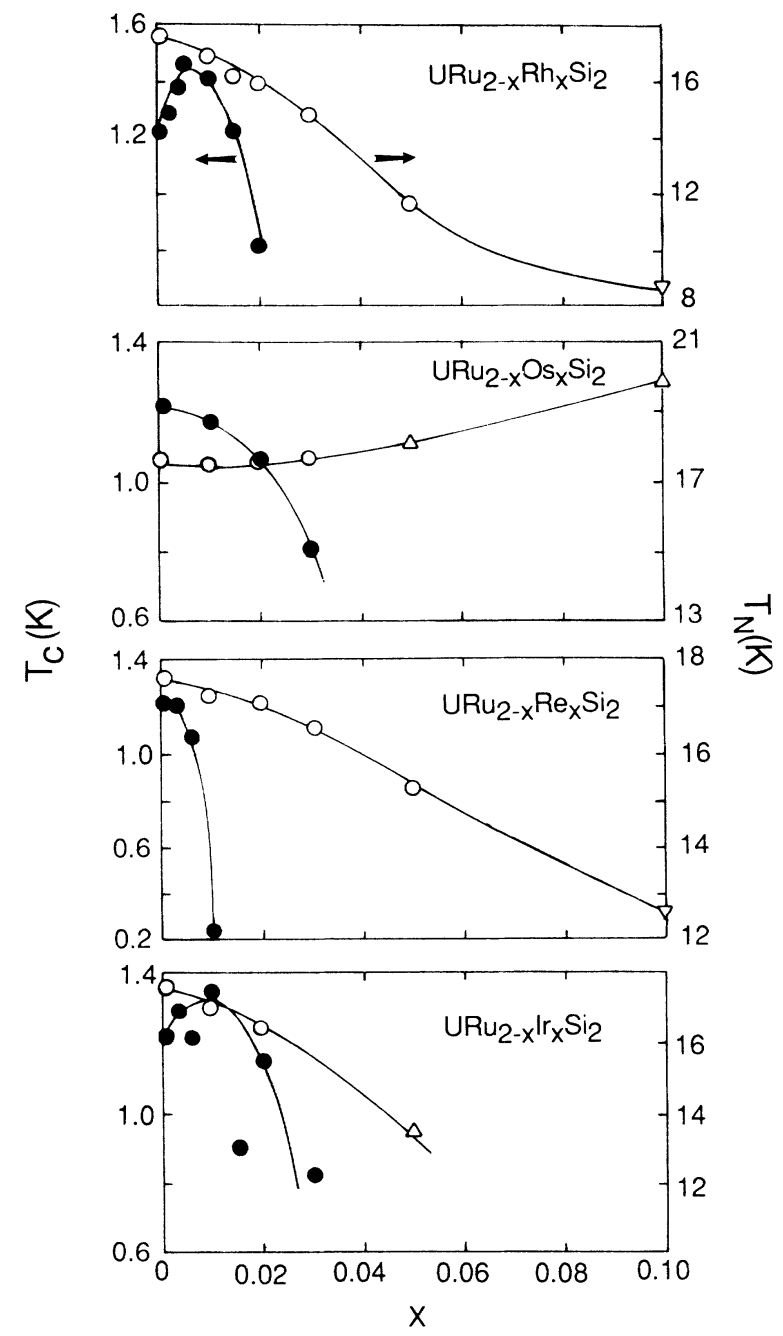

FIG. 8. Impurity concentration $x$ dependence of $T_{N}$ and $T_{c}$ for $\mathrm{URu}_{2-x} M_{x} \mathrm{Si}_{2}(M=\mathrm{Rh}, \mathrm{Os}, \mathrm{Re}$, and $\mathrm{Ir})$. Closed circles represent $T_{c}(x)$ data from ac magnetic-susceptibility measurements. Open circles, triangles, and inverted triangles represent $T_{N}(x)$ data from electrical resistivity, magnetic susceptibility, and specific-heat measurements, respectively. The solid lines are guides to the eye.

compounds rules out a direct overlap of the $f$-electron wave functions so that $f$-level broadening can only occur through hybridization with conduction-electron states. The variation of the number of conduction electrons modifies the degree of hybridization and, in turn, the width of the $f$ level given by $\Gamma=\pi\left\langle V^{2}\right\rangle N(0)$, where $V$ is the hybridization matrix element and $N(0)$ the density of states at the Fermi level (for one spin orientation). Examination of the data reveals that the SDW state in $\mathrm{URu}_{2} \mathrm{Si}_{2}$ is very sensitive to $U 5 f$ ligand hybridization. The increase of the number of conduction electrons via incorporation of $\mathrm{Rh}$ and Ir leads to a depression of $T_{N}$ and a deterioration of the SDW state for $0 \leq x \leq 0.1$, as revealed by the smearing of the anomaly at $T_{N}$. The decrease of conduction-electron density through substitution of $\operatorname{Re}$ or $\mathrm{Tc}$ also leads to a depression of $T_{N}$ accompanied this time by an upturn in the susceptibility below 
$10 \mathrm{~K}$. However, the incorporation of isoelectronic Os atoms, which leaves the conduction-electron density and in turn the degree of hybridization unchanged, is found to increase $T_{N}$ as well as the amplitude of the transition. The increase of the latter may be due to an increase in the SDW energy gap below $T_{N}$.

The inverse correlation between $T_{c}$ and $T_{N}$ displayed by the Ir- and Rh-substituted systems for $0 \leq x<0.01$ and most notably by $\mathrm{URu}_{2-x} \mathrm{Os}_{x} \mathrm{Si}_{2}$ for $0 \leq x<0.1$ is consistent with the picture of two ordered electronic states competing for portions of the Fermi surface. In the $\mathrm{URu}_{2-x} \mathrm{Re}_{x} \mathrm{Si}_{2}$ system, both $T_{c}$ and $T_{N}$ decrease. It is noteworthy that the fraction of the Fermi surface removed by the transition and the energy gap $\Delta$ both decrease with $x$ at a faster rate than $T_{N}$. Assuming that a decrease in $T_{N}$ is associated with a decrease in the fraction of electron states involved in the formation of a SDW rather than a decrease in the coupling constant, then the increase of the electronic specific-heat coefficient $\gamma$ in the $\mathrm{Re}$ - and $\mathrm{Rh}$-doped compounds can be accounted for by a larger density of states available below $T_{N}$ as a consequence of a gap opening over a smaller fraction of the Fermi surface. A mean-field treatment of the competition between superconductivity and CDW formation involving partial gapping of the Fermi surface was dveloped by Bilbro and McMillan ${ }^{23}$ and applied to the pressure dependence of $T_{c}$ and the structural transition temperature of the $A-15$ compounds ${ }^{23}$ and Chevrel phases of the type $\mathrm{Eu}_{1-x} \mathrm{Sn}_{x} \mathrm{Mo}_{6} \mathrm{~S}_{8}{ }^{24}$ The same treatment was extended by Machida ${ }^{25}$ to the SDW case. The proposed relation between $T_{c}$ and $T_{N}$ can be written as $T_{c}^{n} T_{N}^{1-n}=T_{c 0}$, where $T_{c 0}$ is the superconducting transition temperature in the absence of a gap and $n$ is the ungapped fraction of the Fermi surface.

The very small entropy $(\sim 0.01 R \ln 2$ per mole $U)$ associated with the magnetic transition in the $\mathrm{URu}_{2-x} \mathrm{M}_{x} \mathrm{Si}_{2}$ compounds cannot be accounted for by localized spin models, for which the entropy removed by the transition is expected to be $R \ln (2 S+1)$. Overhauser pointed out ${ }^{26}$ that the phase transition from a paramagnetic state to a spin-density wave within the context of a nesting model would have an entropy much smaller than $R \ln (2 S+1)$. In addition, the negligible effect of a magnetic field on the $17.5-\mathrm{K}$ transition in a polycrystalline sample of $\mathrm{URu}_{2} \mathrm{Si}_{2}$ reported by Maple et al. ${ }^{4}$ is similar to the result found in $\mathrm{Cr}$, where the SDW transition temperature is independent of magnetic field to values as high as $16 T^{27}$ However, the same experiment carried out on a single crystal of $\mathrm{URu}_{2} \mathrm{Si}_{2}$ revealed a $2 \%$ decrease of $T_{N}$ when a field of $6 T$ was applied along the $c$ axis. ${ }^{28}$ In a recent review article, Fawcett ${ }^{29}$ pointed out that the spin paramagnetism for a nesting model is the only term in $\chi(T)$ affected by the formation of a SDW and is smaller than the orbital paramagnetism. This is consistent with the small drop in $\chi(T)$ at $T_{N}$ for the $\mathrm{URu}_{2-x} M_{x} \mathrm{Si}_{2}$ alloy systems. The increase of the electrical resistivity with decreasing temperature around $T_{N}$ was first recognized in $\mathrm{Cr}$ by Trego and Mackintosh ${ }^{30}$ as being due to the formation of an energy gap on the nesting part of the Fermi surface, which decreases the effective number of carriers.
It was suggested that the resistivity of $U R u_{2} \mathrm{Si}_{2}$ could be fit by a $T^{2}$ term associated with the low-temperature Fermi-liquid character and an exponential contribution preceeded by a temperature-dependent factor appropriate for an antiferromagnet with energy gap $\Delta .^{3}$ The size of the gap in $\mathrm{URu}_{2} \mathrm{Si}_{2}$, estimated from far-infrared reflectance measurements, ${ }^{31}$ is between 5.6 and $7.5 \mathrm{meV}$, which is comparable to the value of $11 \mathrm{meV}$ inferred from specific-heat measurements, and the shape of its energy dependence is similar to that of the energy gap observed in the SDW state of $\mathrm{Cr}$. However, momentpolarized electron-energy band-structure calculations by Norman et al. ${ }^{32}$ predict that gapping occurs only when the ordered magnetic moment exceeds $1 \mu_{B}$ and, moreover, do not indicate any Fermi-surface nesting.

The thermodynamics of a SDW state closely resembles that of a CDW state. In particular, there is a gap in the single-particle excitation that is described by the BCS equation and a collective mode formed by electron-hole pairs. A Ginzburg-Landau-type microscopic model has been developed by McMillan $^{33}$ to describe the chargedensity wave in $2 \mathrm{H}-\mathrm{TaSe}_{2}$. For a triple-CDW state, the heat-capacity jump is given by

$$
\delta C=14.1 N(0) k_{B}{ }^{2} T_{N},
$$

and the change in susceptibility due to the opening of the gap is

$$
\delta \chi=2.6 N(0) \mu_{B}^{2} \text {. }
$$

In order to compare the model with experiment in $\mathrm{URu}_{2} \mathrm{Si}_{2}$, we first find $N(0) \approx 32$ states/eV-U atom from Eq. (3) and the experimental value $\delta C=5.8 \mathrm{~J} / \mathrm{mol} \mathrm{K}$ for $\mathrm{URu}_{2} \mathrm{Si}_{2}$. Using Eq. (4), the model then yields $\delta \chi \approx 2.7 \times 10^{-3} \mathrm{~cm}^{3} / \mathrm{mol}$, which is approximately four times larger than the experimental value of $0.7 \times 10^{-3}$ $\mathrm{cm}^{3} / \mathrm{mol}$. The model also predicts an energy gap at zero temperature $\Delta \simeq 1.75 k_{B} T_{N}=2.6 \mathrm{meV}$, a factor of 2 smaller than the value estimated from far-infrared reflectance measurements and a factor of 4 smaller than the value estimated from the specific-heat data.

In summary, the effects of various transition-metal substitutions in $\mathrm{URu}_{2} \mathrm{Si}_{2}$ have been investigated. The hybridization between $5 f$ and conduction-electron states is found to have a strong effect on the SDW state, of which the characteristic temperature $T_{N}$ decreases with either increase or decrease of the degree of hybridization. In contrast, isoelectronic Os substitutional atoms induce an increase in $T_{N}$. The particularly large inverse correlation between $T_{N}$ and $T_{c}$ in the Os-doped materials is consistent with two transitions competing for electronic states over the Fermi surface. The increase of $\gamma$, the electronic coefficient of the specific heat, below $T_{N}$ in $x=0.1$ $\mathrm{Re}$ - and $\mathrm{Rh}$-doped materials is consistent with the SDW state forming over a smaller fraction of the Fermi surface.

\section{ACKNOWLEDGMENTS}

We would like to thank B. W. Lee for the lattice parameter calculations and Dr. F. Marsiglio for useful discussions. The research was supported by the U.S. 
Department of Energy under Grant No. DE-FG0386ER45230 and the U.S. National Science Foundation-Low Temperature Physics-Grant No. DMR-84-11839 (YD) at San Diego. One of us (C.R.) would like to acknowledge the support of the Swiss National Science Foundation. Research at Los Alamos National Laboratory was carried out under the auspices of the U.S. Department of Energy.
${ }^{1}$ G. R. Stewart, Rev. Mod. Phys. 56, 755 (1984); P. A. Lee, T. M. Rice, J. W. Serene, L. J. Sham, and J. W. Wilkins, Comments Solid State Phys. 11, 221 (1985).

${ }^{2}$ W. Shlabitz, J. Baumann, B. Pollit, U. Rauchschwalbe, H. M. Mayer, U. Ahlheim, and C. D. Bredl, Z. Phys. B 62, 171 (1986).

${ }^{3}$ T. T. M. Palstra, A. A. Menovsky, J. van den Berg, A. J. Dirkmaat, P. H. Kes, G. J. Nieuwenhuys, and J. A. Mydosh, Phys. Rev. Lett. 55, 2727 (1985).

${ }^{4}$ M. B. Maple, J. W. Chen, Y. Dalichaouch, T. Kohara, C. Rossel, M. S. Torikachvili, M. W. McElfresh, and J. D. Thompson, Phys. Rev. Lett. 56, 185 (1986).

${ }^{5}$ G. Aeppli, E. Bucher, C. Broholm, J. K. Kjems, J. Baumann, and J. Hufnagl, Phys. Rev. Lett. 60, 615 (1988).

${ }^{6}$ Y. J. Uemura, W. J. Kossler, X. H. Yu, H. E. Schone, J. R. Kempton, C. E. Stronach, S. Barth, F. N. Gygax, B. Hitti, A. Schenck, C. Baines, W. F. Lankford, Y. Onuki, T. Komatsubara, Physica C 153-155, 455 (1988).

${ }^{7}$ See, for example, Superconductivity in Ternary Compounds II, edited by M. B. Maple and $\emptyset$. Fisher (Springer-Verlag, Berlin, 1982).

${ }^{8}$ H. R. Ott, H. Rudigier, P. Delsing, and Z. Fisk, Phys. Rev. Lett. 52, 1551 (1984).

${ }^{9}$ Z. Fisk, G. R. Stewart, J. O. Willis, H. R. Ott, and F. Hulliger, Phys. Rev. B 30, 6360 (1984).

${ }^{10}$ H. J. van Daal, K. H. J. Buschow, P. B. van Aken, and M. H. van Maaren, Phys. Rev. Lett. 34, 1457 (1975); H. R. Ott, H. Rudigier, E. Felder, Z. Fisk, and B. Batlogg, ibid. 55, 1595 (1985).

${ }^{11}$ A. P. Ramirez, B. Batlogg, E. Bucher, and A. S. Cooper, Phys. Rev. Lett. 57, 1072 (1986); G. R. Stewart, A. L. Giorgi, J. O. Willis, and J. O'Rourke, Phys. Rev. B 34, 4629 (1986).

${ }^{12}$ A. de Visser, J. C. P. Klaasse, M. van Sprang, J. J. M. Franse, A. A. Menovsky, and T. T. M. Palstra, J. Magn. Magn. Mater. 54-57, 375 (1986); R. Verhoef, A. de Visser, A. A. Menovsky, A. J. Riemersma, and J. J. M. Franse, Physica 142B, 11 (1986).

${ }^{13}$ C. Broholm, J. K. Kjems, W. J. L. Buyers, P. Matthews, T. T. M. Palstra, A. A. Menovsky, and J. A. Mydosh, Phys. Rev. Lett. 58, 1467 (1987).
${ }^{14}$ T. Kohara, Y. Kohori, K. Asayama, Y. Kitaoka, M. B. Maple, and M. S. Torikachvili, Jpn. J. Appl. Phys. 26, Suppl. 263, 1247 (1987).

${ }^{15}$ J. Schoenes, C. Schönenberger, J. J. M. Franse, and A. A. Menovsky, Phys. Rev. B 35, 5375 (1987).

${ }^{16}$ M. F. Hundley, L. C. Bourne, A. Zettl, C. Rossel, and M. B. Maple, Solid State Commun. 62, 603 (1987).

${ }^{17}$ U. Walter, C.-K. Loong, M. Loewenhaupt, and W. Schlabitz, Phys. Rev. B 33, 7875 (1986).

${ }^{18}$ Z. Ban and M. Sikirica, Acta Crystallogr. 18, 594 (1965).

${ }^{19}$ L. G. Akselrud, Ja. P. Jarmoljuk, and E. I. Gladyshevskij, Dok. Akad. Nauk SSSR, 40, 359 (1978).

${ }^{20} \mathrm{Because}$ of the presence of macroscopic cracks in the polycrystalline samples, the absolute electrical resistivity could not be calculated accurately.

${ }^{21}$ Y. Dalichaouch, M. B. Maple, M. S. Torikachvili, and A. L. Giorgi, Phys. Rev. B 39, 2423 (1989).

${ }^{22}$ W. B. Pearson, The Crystal Chemistry and Physics of Metals and Alloys (Wiley, New York, 1972).

${ }^{23}$ G. Bilbro and W. L. McMillan, Phys. Rev. B 14, 1887 (1976).

${ }^{24}$ R. C. Lacoe, S. A. Woolf, P. M. Chaikin, C. Y. Huang, and H. L. Luo, Phys. Rev. Lett. 48, 1212 (1982).

${ }^{25}$ K. Machida and M. Kato, Phys. Rev. Lett. 58, 1986 (1987).

${ }^{26}$ A. W. Overhauser, Phys. Rev. 128, 1437 (1962).

${ }^{27}$ R. L. Melcher and W. D. Wallace, Solid State Commun. 8, 1535 (1970); Z. Barak, E. Fawcett, D. Feder, G. Lorincz, and M. B. Walker, J. Phys. F 11, 915 (1981).

${ }^{28}$ T. Fukase, Y. Koike, T. Nakanomyo, Y. Shiokawa, A. A. Menovsky, J. A. Mydosh, and P. H. Kes, Jpn. J. Appl. Phys. 26 Suppl. 26-3, 1249 (1987).

${ }^{29}$ E. Fawcett, Rev. Mod. Phys. 60, 209 (1988), and references therein.

${ }^{30}$ A. L. Trego and A. R. Mackintosh, Phys. Rev. 166, 495 (1968).

${ }^{31}$ D. A. Bonn, J. D. Garret, and T. Timusk, Phys. Rev. Lett. 61, 1305 (1988).

${ }^{32}$ M. R. Norman, T. Ogushi, and A. J. Freeman, Phys. Rev. B 38, 11193 (1988).

${ }^{33}$ W. L. McMillan, Phys. Rev. B 16, 643 (1977). 